\title{
Raman, infrared and optical spectra of the spin-Peierls compound $\mathrm{NaV}_{2} \mathrm{O}_{5}$
}

\author{
S. A. Golubchik ${ }^{a}$, M. Isobe ${ }^{b}$, A. N. $\operatorname{Ivlev}^{a}$, B. N. Mavrin ${ }^{a}$, M. N. Popova ${ }^{a}$ 円, A. B. Sushkov ${ }^{a}$, \\ Yu. Ueda ${ }^{b}$, and A. N. Vasil'ev ${ }^{c}$,
}

${ }^{a}$ Institute of Spectroscopy of RAS, Troitsk, 142092, Russia

${ }^{b}$ Institute for Solid State Physics, The University of Tokyo

7-22-1 Roppongi, Minato-ku, Tokyo 106, Japan

${ }^{c}$ Physics Department, Moscow State University

119899 Moscow, Russia

Keywords: phonons, Raman spectra, FIR transmission, $d$-electrons.

We have measured polarized spectra of Raman scattering, infrared and optical transmission of $\mathrm{NaV}_{2} \mathrm{O}_{5}$ single crystals above the temperature of the spin-Peierls transition $T_{s p}=35 \mathrm{~K}$. Some of the far-infrared (FIR) phonon lines are strongly asymmetric, due to the spin-phonon interaction. In addition to the phonon lines, a broad band was observed in the $c(a a) \bar{c}$ Raman spectrum and in the $\mathbf{E} \|$ a FIR transmission spectrum. A possible origin of these bands is discussed. The absorption band at $10000 \mathrm{~cm}^{-1}(1.25 \mathrm{eV})$ is attributed to vanadium $d-d$ electronic transitions while the absorption edge above $3 \mathrm{eV}$ is supposed to correspond to the onset of charge-transfer transitions. 


\section{INTRODUCTION}

$\mathrm{NaV}_{2} \mathrm{O}_{5}$ crystallizes in an orthorhombic cell with space group $P 2_{1} m n$ and two formula units in the primitive cell [1]. All the atoms occupy twofold positions $2 a$ having the symmetry $C_{s}$. There are magnetic chains of the $\mathrm{V}^{4+} \mathrm{O}_{5}(S=1 / 2)$ square pyramids in the structure isolated by nonmagnetic chains of the $\mathrm{V}^{5+} \mathrm{O}_{5}(\mathrm{~S}=0)$ pyramids. Both binds of chains are oriented along the b-axis. These chains form layers while sodium ions lie between the layers. Above $35 \mathrm{~K}$, the magnetic susceptibility of $\mathrm{NaV}_{2} \mathrm{O}_{5}$ is well described by the equation for the $S=1 / 2$ 1-D antiferromagnetic (AF) Heisenberg linear chain with nearest neighbor interaction $\mathcal{H}=\sum_{i} \mathrm{JS}_{i} \mathbf{S}_{i+1}$ and exchange constant $J=560 \mathrm{~K}$ [2]. It has been shown recently [2]- [at that at $T_{s p}=35 \mathrm{~K} \mathrm{NaV}_{2} \mathrm{O}_{5}$ undergoes the spin-Peierls transition, namely, the spin-singlet nonmagnetic ground state is formed, simultaneously with a dimerization of the atoms along the chains. As a result of the dimerization, an energy gap $\Delta$ opens in the magnetic excitation spectrum $\left(\Delta \simeq 9.8 \mathrm{meV}=79 \mathrm{~cm}^{-1}\right.$ in the case of $\mathrm{NaV}_{2} \mathrm{O}_{5}$ [3] $)$. The spin-Peierls transition is mediated by the interaction between an isotropic 1-D $S=1 / 2 \mathrm{AF}$ spin system and a 3-D phonon field. In the weak coupling limit, the BCS-type relation $2 \Delta \simeq 3.53 k T_{s p}$ must be valid [5]. All the spin-Peierls compounds studied before obeyed the mentioned relation rather well. On the contrary, $\mathrm{NaV}_{2} \mathrm{O}_{5}$, the compound with the highest known $T_{s p}$ demonstrates a strong deviation from this relation $\left(2 \Delta \simeq 6.4 k T_{s p}\right.$ for $\left.\mathrm{NaV}_{2} \mathrm{O}_{5}\right)$ which implies that the spin-phonon coupling is not weak. Manifestations of this coupling in the far-infrared (FIR) spectra of $\mathrm{NaV}_{2} \mathrm{O}_{5}$ have recently been reported in Ref.[6]. To better understand peculiarities of the spin-Peierls transition in $\mathrm{NaV}_{2} \mathrm{O}_{5}$ it is important to know its spin and lattice dynamics and energy spectrum.

The present paper is devoted, mainly, to a thorough study of the phonon spectrum of $\mathrm{NaV}_{2} \mathrm{O}_{5}$ in the uniform phase (at $T>T_{s p}$ ) by means of polarized Raman scattering and far-infrared absorption. Preliminary data on the electronic spectrum are also presented.

\section{EXPERIMENTAL}

The $\mathrm{NaV}_{2} \mathrm{O}_{5}$ single crystals used in this study were obtained as described in Ref. [2, 3] and had dimensions of approximately $1 \times 4 \times 0.5 \mathrm{~mm}^{3}$ along the $a^{-}, b$-, and $c$-axes. The orientation of the crystals and lattice constants $(a=11.318 \AA, b=3.611 \AA, c=4.797 \AA)$ were determined by X-ray method. We used, in particular, the samples studied before by the EPR and magnetic susceptibility measurements [4]. The platelets with the thickness of $0.4-40 \mu \mathrm{m}$ along the $c$-axis were prepared for FIR and optical measurements.

Raman spectra were excited at room temperature by the $514 \mathrm{~nm}$ and $488 \mathrm{~nm}$ lines of an Ar-ion laser in backscattering geometries, dispersed by a home-made triple spectrograph and recorded using a multichannel system consisting of an image intensifier tube with a multichannel plate and a vidicon.

Polarized transmission measurements were made in the spectral range $50-30000 \mathrm{~cm}^{-1}$ (3 meV-3.7 eV) with the resolution ranging from 0.05 to $16 \mathrm{~cm}^{-1}$ at the temperatures $40-$ $300 \mathrm{~K}$ using a Fourier transform spectrometer BOMEM DA3.002 (for the spectral range 50-20000 $\mathrm{cm}^{-1}$ ) and a grating spectrometer Specord M400 (for the spectral range 15000$\left.30000 \mathrm{~cm}^{-1}\right)$.

\section{RESULTS AND DISCUSSION}

A. RAMAN AND INFRARED SPECTRA 
The factor group analysis gives the following vibrational representation for $\mathrm{NaV}_{2} \mathrm{O}_{5}$ :

$$
\Gamma=16 A_{1}+8 A_{2}+8 B_{1}+16 B_{2}
$$

(Here, the irreducible representations refer to the standard setting for $P m n 2_{1}$ space group where the $x$-, $y$-, and $z$-axes are directed along the $b$-, $c$ - and $a$-axes, respectively). Subtracting three acoustical modes $\left(A_{1}+B_{1}+B_{2}\right)$, we get 45 optical modes. All the optical modes are Raman active. The diagonal components $a a, b b, c c$ of the Raman scattering tensor which manifest themselves for the parallel polarizations $\mathbf{E}_{\mathbf{i}} \| \mathbf{E}_{\mathbf{s}}$ of the incident and scattered light are nonzero only for the $A_{1}$ modes. In the $\mathbf{E}_{\mathbf{i}} \perp \mathbf{E}_{\mathbf{s}}$ geometries another modes are active, namely, $a b, a c$ and $b c$ components of the scattering tensor are nonzero for the $B_{1}, B_{2}$ and $A_{2}$ modes, respectively. In the FIR absorption, $A_{2}$ modes are silent, $A_{1}, B_{1}$, and $B_{2}$ modes are active for the polarization of the incident light $\mathbf{E}\|\mathbf{a}, \mathbf{E}\| \mathbf{b}$ and $\mathbf{E} \| \mathbf{c}$, respectively. In our FIR experiment the wave vector of the incident light was parallel to the $c$-axis, so we could observe $A_{1}$ modes (for $\mathbf{E} \| \mathbf{a}$ ) and $B_{1}$ modes (for $\mathbf{E} \| \mathbf{b}$ ).

Figs. 1-3 show polarized Raman spectra of $\mathrm{NaV}_{2} \mathrm{O}_{5}$. Fig. 1 presents the spectra for $\mathbf{E}_{\mathbf{i}} \|$ $\mathbf{E}_{\mathbf{s}}$, in other words, $A_{1}$ modes. Raman spectra turned out to be the most intense in the geometry $a(c c) \bar{a}$ where longitudinal optical (LO) $A_{1}$ modes are active. In the two other geometries, $c(b b) \bar{c}$ and $c(a a) \bar{c}$, the wave vector of a phonon is perpendicular to the $a$-axis and, thus, transverse optical (TO) $A_{1}$ modes must be active. Nevertheless, the spectra in these two geometries differ markedly, which points to the considerable anisotropy of the structure in the directions of the $a$ - and $b$-axes ( $b$-axis is parallel to the chains of $\mathrm{VO}_{5}$ pyramids). Frequencies of all the observed Raman modes are collected in table 1. Positions of the five observed LO $A_{1}$ modes are very close to the positions of some TO $A_{1}$ modes, indicating small LO-TO splittings and, hence, small oscillator strengths of these modes.

Besides the phonon lines, we observed a broad band in the $c(a a) \bar{c}$ spectrum, with a maximum near $600 \mathrm{~cm}^{-1}$, extending from 200 to $900 \mathrm{~cm}^{-1}$. Since this band appears under both $514.5 \mathrm{~nm}$ and $488 \mathrm{~nm}$ excitation (see fig. 2), we conclude that it originates from the Raman scattering process. According to preliminary data, the broad band near $600 \mathrm{~cm}^{-1}$ is present down to liquid helium temperatures. Its interpretation is not yet clear. It follows from the data on the magnetic susceptibility of $\mathrm{NaV}_{2} \mathrm{O}_{5}$, which has a broad maximum around the room temperature, that a short range order (SRO) and, hence, (damped) magnetic excitations are present in the $\mathrm{V}^{4+} \mathrm{O}_{5}$ magnetic chains even at room temperature. One could expect to observe two-mode magnetic scattering in $\mathrm{NaV}_{2} \mathrm{O}_{5}$. Such a scattering has been found in another spin-Peierls compound $\mathrm{CuGeO}_{3}$ at the temperatures when $\mathrm{SRO}$ is preserved [8]. However, such a scattering should have a maximum intensity for parallel polarization of incoming and scattered light, direction of polarization being along the magnetic chains [8] (that is, in (bb) geometry), as is not the case experimentally. Therefore, it is necessary to consider another interpretation of this band, for example, two-phonon processes or electronic Raman scattering to a low lying d-level of the $\mathrm{V}^{4+}$ ion. We shall return to this point below.

For crossed polarization of the incident and scattered light, the spectra turned out to be much weaker than for parallel polarization (see fig. 3). From the eight predicted $A_{2}$ modes we could find only five in the $a(c b) a$ geometry. They are listed in table 1 . Furthermore, we can not exclude that the lines at 89 and $970 \mathrm{~cm}^{-1}$ appear due to leakage of very intense $(c c)$ components of the Raman tensor.

From the fifteen TO $B_{2}$ modes we were able to find only seven in the $b(a c) \bar{b}$ geometry. In 
Even less number of Raman lines was seen in the $c(a b) \bar{c}$ geometry where transverse $B_{1}$ modes should be active. From the expected seven $B_{1}$ modes we have found only three. The broad band displayed above for the $c(a a) \bar{c}$ geometry is present also for the crossed polarization, though slightly displaced and weak in intensity.

Polarized infrared transmittance spectra of $\mathrm{NaV}_{2} \mathrm{O}_{5}$ in the uniform phase are given in figs. 4,5. In fig. 4 we show the spectral range $50-2500 \mathrm{~cm}^{-1}$ while in fig. 5 the far-infrared region is displayed in detail. Peaks in transmittance of a thin crystal are located at the TO phonon frequencies. To study modes with different oscillator strengths we used samples of different thickness. At room temperature, we were able to detect only three $A_{1}$ modes and three $B_{1}$ modes. Below $200 \mathrm{~K}$ several more modes became observable due to the narrowing of their lines. The TO phonon frequencies found from the polarized FIR transmittance spectra are listed in table 1. Some of the phonon lines show a pronounced asymmetry (see fig. 5). It should be mentioned here that the thinnest sample (3) used was not uniform in thickness, so in its case the lineshapes were not correct. The $B_{1}$ mode $215 \mathrm{~cm}^{-1}$ and the $A_{1}$ mode $145 \mathrm{~cm}^{-1}$ have high frequency tails. The lowest frequency $A_{1}$ mode $\left(\nu=90 \mathrm{~cm}^{-1}\right)$ has a shape characteristic for Fano type resonance (the resonance between a descrete level and a continuum of states (77). We suppose that asymmetric lineshapes are due to the spin-phonon coupling that drives the system into a dimerized phase (see also Ref.[6]).

More thick samples reveal, in addition to the phonon lines, a broad $\mathbf{E} \|$ a polarized absorption continuum of a complex shape extending from very low frequencies (below $50 \mathrm{~cm}^{-1}$ ) to about $1500 \mathrm{~cm}^{-1}$ (see fig. 4). We suppose that at least a part of this continuum arises from the two-mode magnetic absorption. It follows from the symmetry consideration that in the case of $\mathrm{NaV}_{2} \mathrm{O}_{5}$ such an absorption should have a maximum intensity in the $\mathbf{E} \| \mathbf{a}$ polarization [9, 6].

The phonon frequencies extracted from our Raman and FIR measurements are summarized in table 1. Not all the predicted modes have been found and that does not allow to carry out calculations of the lattice dynamics and to draw ionic displacement patterns for the observed modes. Nevertheless, we present below some considerations concerning a character of the observed vibrational modes.

The crystal structure of $\mathrm{NaV}_{2} \mathrm{O}_{5}$ consists of layers of the $\mathrm{VO}_{5}$ pyramids interconnected by their edges or corners [1, 2]. Bonds between vanadium and oxygen atoms are predominantly covalent. Weak interlayer coupling is due to Van-der-Vaals interaction between apical oxygens of the pyramids in neighboring layers. Sodium atoms are situated between the $\mathrm{VO}_{5}$ pyramids within a layer and form ionic bonds with nearest oxygens. Usually, the intensity of Raman scattering from vibrations of ionically bound atoms is small as compared to the intensity of scattering from vibrations of atoms with covalent bonds. On the contrary, ionic vibrations should be strong in the FIR spectra. The strongest IR lines $530 \mathrm{~cm}^{-1}\left(\mathrm{~A}_{1}\right)$ and $594 \mathrm{~cm}^{-1}\left(B_{1}\right)$ come, probably, from the $\mathrm{Na}-\mathrm{O}$ ionic vibrations.

The primitive cell of the $\mathrm{NaV}_{2} \mathrm{O}_{5}$ crystal contains only one layer. Therefore, interlayer vibrations must be absent and all the observed modes must refer to the intralayer vibrations. The high frequency modes (in the region of $500 \mathrm{~cm}^{-1}$ and higher) most probably originate from dominantly oxygen vibrations in $\mathrm{VO}_{5}$ pyramids while modes below $100 \mathrm{~cm}^{-1}$ dominantly come from vibrations of vanadium atoms. In the intermediate frequency region both types of atoms participate in vibrations. According to the structural data [1], the distance between vanadium and apical oxygen is the smallest one among the $\mathrm{V}$-O distances in the 
while the other V-O distances are $1.89-1.98 \AA$ for the first kind of pyramids and $1.76-1.98 \AA$ for the second one. On that basis we suppose that the highest frequency mode at $970 \mathrm{~cm}^{-1}$ well separated from the other modes originates most probably from vibrations of the apical oxygens.

\section{B. OPTICAL SPECTRA}

The region of phonon frequencies is followed by a transparency window up to $5000 \mathrm{~cm}^{-1}$. We have determined the refractive indexes $n_{a}$ and $n_{b}$ of $\mathrm{NaV}_{2} \mathrm{O}_{5}$ crystal for $\mathbf{E} \|$ a and $\mathbf{E} \| \mathbf{b}$, from the interference patterns observed within this window, according to the relation $\Delta=1 /(2 d n)$ (here, $\Delta$ is the period of interference, $d$ - the thickness of the crystal). We obtained the following average values over the spectral interval from $2000 \mathrm{~cm}^{-1}$ to $4000 \mathrm{~cm}^{-1}$ : $n_{a} / n_{b}=1.38 \pm 6 \%, n_{a}=3.7 \pm 0.9, n_{b}=2.7 \pm 0.7$.

Above $0.62 \mathrm{eV}\left(5000 \mathrm{~cm}^{-1}\right)$, a broad complex absorption band is seen (fig. 5) followed by a transmittance window in the blue region of spectrum and an anisotropic absorption edge in the ultraviolet (uv). By the analogy with vanadium oxydes studied before (see, e.g, [10]), we ascribe this band to $d-d$ transitions of $\mathrm{V}^{4+}$ ions but the uv absorption edge - to the onset of charge transfer transitions. In fig. 7 , we propose a simplified scheme to account for the observed $d-d$ electronic transitions in $\mathrm{NaV}_{2} \mathrm{O}_{5}$. Fivefold orbitally degenerate energy level of a $d$-electron in a free $\mathrm{V}^{4+}$ ion is split into three $A^{\prime}$ and two $A^{\prime \prime}$ levels by the crystal field of $C_{s}$ symmetry. There are two equivalent $\mathrm{V}^{4+}$ ions in the unit cell (they are situated in the neighboring $\mathrm{V}^{4+}-\mathrm{O}$ chains). As a result, each excited electronic energy level $A^{\prime}$ or $A^{\prime \prime}$ of the $\mathrm{V}^{4+}$ local symmetry group $C_{s}(y z)$ gives rise to $A_{1}-B_{2}$ or $A_{2}-B_{1}$, respectively, Davydov doublets of the crystal factor group $C_{2 v}(z)$. Electric dipole optical transitions from the fully symmetrical $\left(A_{1}\right)$ ground crystal state are allowed to the $A_{1}, B_{1}$ and $B_{2}$ levels in $\mathbf{E} \| \mathbf{a}$, $\mathbf{E}\|\mathbf{b}, \mathbf{E}\| \mathbf{c}$ polarization, respectively. These selection rules are violated by the spin-orbit coupling which converts each level into a $E_{1 / 2}$ Kramers doublet. $E_{1 / 2} \rightarrow E_{1 / 2}$ transitions are allowed for all the polarizations of the incident light and all the components of the Raman scattering tensor. However, the transitions obeying the above mentioned selection rules should dominate.

The Davydov splitting is governed by the interaction between two $\mathrm{V}^{4+}$ ions in the neighboring chains, while the width of the total $E(k)$ crystal energy band depends on the coupling between neighboring $\mathrm{V}^{4+}$ ions in the same chain. As the interaction within the chain is much stronger than the one between the chains, the Davydov splitting must be much smaller than the width of the optical absorption bands.

Possibly, the lowest Davydov pair of levels lies at about $600 \mathrm{~cm}^{-1}$ and is responsible for a part of the observed continuum in FIR absorption and for the broad band in Raman spectra, while the next three Davydov pairs lie in the energy range between 0.74 and $2.1 \mathrm{eV}$ and lead to the broad absorption band in this spectral region.

Acknowledgement

We are grateful to G.N.Zhizhin for a support. This work was made possible in part by Grants N95-02-03796-a, N96-02-18114-a and N96-02-19474-a from the Russian Fund for Fundamental Research.

\section{References}


[1] A.Carpy and J.Galy, Acta Crystallogr. B 31 (1975) 1481.

[2] M.Isobe and Y.Ueda, J. Phys. Soc. Jap. 65 (1996) 1178.

[3] Y.Fujii, H.Nakao, T.Iosihama, M.Nishi, K.Nakajima, K.Kakurai, M.Isobe, Yu.Ueda and H.Sawa, Tech. Report ISSP, Ser. A, No.3171, (1996).

[4] A.N.Vasil'ev, A.I.Smirnov, M.Isobe and Y.Ueda, Electron spin resonance in spin-Peierls compound $\mathrm{NaV}_{2} \mathrm{O}_{5}$, submitted to Phys. Rev. B.

[5] A.I.Buzdin and L.N.Bulaevskii, Uspekhi Fizicheskikh Nauk 131 (1980) 495.

[6] M.N.Popova, A.B.Sushkov, A.N.Vasil'ev, M.Isobe, and Yu.Ueda, Pis'ma v ZhETF 65 (1997) 711.

[7] U.Fano, Phys. Rev. B 124 (1961) 1866.

[8] P. van Loosdrecht, J.P.Boucher, G.Martinez, G.Dhalenne and A.Revcolevschi, Phys. Rev. Lett. 76 (1996) 311.

[9] T.Moriya, J. Appl. Phys. 39 (1968) 1042.

[10] W. van Hove, P.Clauws, and J.Vennik, Sol. St. Comm. 33 (1980) 11.

Figure captions

Fig. 1. Room-temperature Raman spectra of $\mathrm{NaV}_{2} \mathrm{O}_{5}$ for parallel polarizations of the incident and scattered light.

Fig. 2. Room-temperature Raman spectra of $\mathrm{NaV}_{2} \mathrm{O}_{5}$ in the $c(a a) \bar{c}$ geometry at $514.5 \mathrm{~nm}$ and $488 \mathrm{~nm}$ excitation.

Fig. 3. Room-temperature Raman spectra of $\mathrm{NaV}_{2} \mathrm{O}_{5}$ for crossed polarizations of the incident and scattered light.

Fig. 4. Polarized IR transmittance spectra of different samples of $\mathrm{NaV}_{2} \mathrm{O}_{5}$. (1) The sample of the thickness $d \simeq 13 \mu \mathrm{m}$. Interference within the sample is seen; $T=300 \mathrm{~K}$. (2) $d \simeq 0.8 \mu \mathrm{m}$, the sample on a scotch tape, $T=300 K$. (3) $d \simeq 0.4 \mu \mathrm{m}$, the sample on a scotch tape; $T=40 \mathrm{~K}$. Arrows indicate phonon frequencies.

Fig. 5. Polarized FIR transmittance spectra of $\mathrm{NaV}_{2} \mathrm{O}_{5}$. (1) $d \simeq 13 \mu \mathrm{m}, T_{1}=300 \mathrm{~K}$ (solid line), $T_{2}=40 \mathrm{~K}$ (dashed line); (2) $d \simeq 0.8 \mu \mathrm{m}, T=300 \mathrm{~K} ;(3) d \simeq 0.4 \mu \mathrm{m}, T=40 \mathrm{~K}$.

Fig. 6. Room-temperature optical absorption spectrum of $\mathrm{NaV}_{2} \mathrm{O}_{5}$ single crystal. 


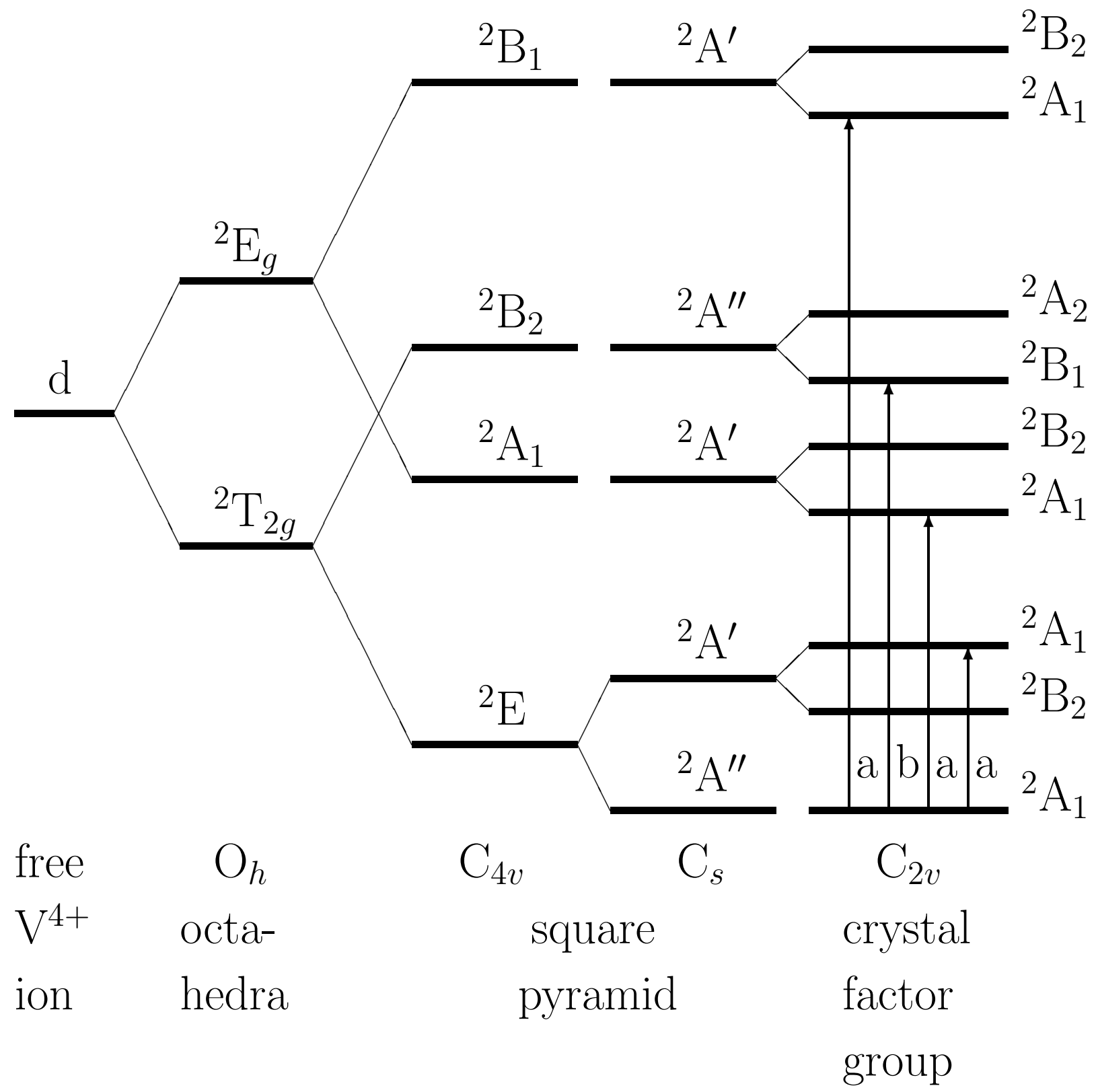

Fig. 7. Scheme of the $d$-level splitting in a crystal. Allowed optical transitions are shown only for the $\mathbf{E} \| \mathbf{a}$ and $\mathbf{E} \| \mathbf{b}$ polarizations available in our $\mathbf{k} \| \mathbf{c}$ experimental geometry. 
Table 1: Frequencies of the vibrational modes observed in the Raman and far-infrared transmission spectra of $\mathrm{NaV}_{2} \mathrm{O}_{5}$

\begin{tabular}{|c|c|c|c|c|}
\hline \multirow{3}{*}{ mode } & \multicolumn{3}{|c|}{$\omega_{T O}, \mathrm{~cm}^{-1}$} & \multirow{3}{*}{$\begin{array}{c}\omega_{L O}, \mathrm{~cm}^{-1} \\
\text { Raman } \\
300 \mathrm{~K}\end{array}$} \\
\hline & \multicolumn{2}{|c|}{ FIR transmission } & \multirow{2}{*}{$\begin{array}{c}\text { Raman } \\
300 \mathrm{~K}\end{array}$} & \\
\hline & $40 \mathrm{~K}$ & $300 \mathrm{~K}$ & & \\
\hline \multirow[t]{13}{*}{$\mathrm{A}_{1}$} & 90 & & 89 & 89 \\
\hline & 140 & 145 & & \\
\hline & & & 175 & 175 \\
\hline & & & 215 & 225 \\
\hline & & & 231 & \\
\hline & 254 & 251 & 254 & \\
\hline & & & 300 & \\
\hline & & & 415 & 419 \\
\hline & & & 421 & \\
\hline & & & 435 & \\
\hline & & & 449 & \\
\hline & 531 & 526 & 531 & \\
\hline & & & 970 & 970 \\
\hline \multirow[t]{6}{*}{$\mathrm{B}_{1}$} & 178 & 175 & 172 & \\
\hline & 215 & & & \\
\hline & 225 & & & \\
\hline & & & 289 & \\
\hline & 371 & 367 & & \\
\hline & 594 & 587 & & \\
\hline
\end{tabular}

$\mathrm{A}_{2}$

89

260

365

685

970

$\mathrm{B}_{2}$ 